\title{
Editorial 2/2009
}

\section{Florence Hartmann-Vareilles • Pilar Núñez Ruiz}

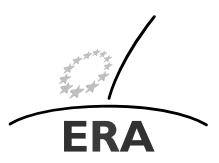

(C) ERA 2009

EUROPÄISCHE RECHTSAKADEMIE ACADEMY OF EUROPEAN LAW ACADEMIE DE DROIT EUROPEEN ACCADEMIA DI DIRITTO EUROPEO
TRIER - TREYES - TREYIRI

Ce deuxième numéro d'ERA Forum 2009, qui se penche sur différentes matières relevant du champ de compétence de la section de droit des affaires, nous permet de mettre en exergue les sept années d'existence de cette section au sein du département des programmes de l'Académie de Droit européen de Trèves (ci-après ERA).

Au début de l'année 2002, l'ERA a mis en place une section spécialisée sous le titre «Droit des affaires » dont le nombre de matières a sensiblement augmenté ces dernières années afin de répondre aux développements du droit communautaire.

Aujourd'hui, le nombre de matières couvert par la section II, droit des affaires, s'est encore élargi de sujets dont l'intérêt s'est manifesté plus tard, tel que le droit des denrées alimentaires, le droit de la santé et le droit pharmaceutique, le droit des aides d'État, le droit du commerce international, le droit bancaire et le droit des douanes.

Ce numéro qui reflète cette richesse thématique et éclectique nous a amené à regrouper les articles en trois différents groupes : la protection du consommateur (assurances ; marques ; brevets, pesticides et le droit des denrées alimentaires); le droit de la concurrence (les aides d'états) et la fiscalité (l'impact dans les opérations transfrontalières).

L'abondance du contenu de la section 2 se traduit par une fertilité de séminaires, dont certains sont devenus des évènements réguliers organisés annuellement par l'ERA, ayant pour finalité de porter à la connaissance des praticiens les récents développements du droit européen des affaires. Les articles présentés dans ce numéro

F. Hartmann-Vareilles $(\bowtie) \cdot$ P. Núñez Ruiz

Droit européen des affaires, Académie de Droit européen, Metzer Allee 4, 54295 Trier, Germany

e-mail: fhartmann@era.int

P. Núñez Ruiz

e-mail: pnunez@era.int 
reprennent les contributions faites lors de plusieurs conférences ouvertes organisées par l'Académie de droit européen de Trèves entre le deuxième semestre 2008 et le premier semestre 2009.

\section{La protection du consommateur}

Une première série d'articles peut être analysée selon une approche horizontale liée à la prise en compte croissante de l'intérêt et de la protection du consommateur dans les politiques communautaires. Le droit européen de la consommation a débuté dans les années 1975, mais la réalisation du marché unique en 1993 a ouvert une phase nouvelle de l'action communautaire et correspond à la prise de conscience de la Commission européenne du fait qu'il ne suffisait pas d'assurer la libre circulation des marchandises et des services, mais qu'il fallait aussi veiller à ce que les consommateurs aient suffisamment confiance en ces produits. La réalisation de ces objectifs s'est faite notamment par une politique communautaire de promotion et de protection des intérêts des consommateurs, englobant le droit à la protection de la santé et de la sécurité, le droit à l'information et l'accès à la justice. Ce dernier objectif, qui donne la faculté au consommateur d'obtenir réparation des dommages encourus et le droit d'être entendu, fait partie des mesures destinées à résoudre les conflits qui peuvent naître entre commerçants ou producteurs et consommateurs.

En dehors de la méthode contentieuse classique de résolution des conflits, il existe des possibilités non-contentieuses couramment utilisées dans certains domaines du droit commercial, comme celui des assurances, ainsi que Christian Duve, Associé du Cabinet Freshfields Bruckhaus Deringer, l'expose dans son article sur « Recent developments in legislation and jurisprudence in the area of European Insurance Law Avoiding litigation : a practitioner's approach » où il approche concrètement la méthode de résolution alternative des conflits dans le cadre spécifique des assurances. Exportée des Etats-Unis, cette méthode rencontre une popularité croissante en Europe en raison des avantages qu'elle offre par rapport au contentieux classique : économie de temps et d'argent, confidentialité, degré élevé d'expertise juridique offerte par les experts désignés, etc. Si elle peut prendre différentes formes (négociation, médiation, conciliation, arbitrage, etc.), elle peut cependant ne pas être adaptée à tous les conflits entre assurés, assureurs et tiers. Cette pratique connaît un essor important en Allemagne où notamment, l'association allemande des assureurs (Gesamtverband der Deutschen Versicherungswirtschaft) a introduit en 2001 le concept innovateur de médiateur en assurance (Versicherungsombudsmann). L'auteur conclut cependant que si ce mode alternatif de résolution des conflits représente un certain nombre d'avantages incontestables, il ne peut être considéré que comme une option et non comme un substitut au contentieux.

Les priorités de l'actuelle politique communautaire dans le domaine de la protection des consommateurs telles qu'énoncées dans la Communication de la Commission du 13 mars 2007 portant sur la «Stratégie communautaire en matière de politique des consommateurs pour la période 2007-2013» se concentrent sur l'amélioration de la réglementation, le contrôle de l'application des lois et des voies de recours, la surveillance du marché par un renforcement des outils existants, et l'information des 
consommateurs, ainsi que le placement du consommateur au centre des politiques communautaires (telles que la santé, les entreprises, l'industrie, l'environnement ou les transports).

La propriété intellectuelle et industrielle est l'une des politiques où incontestablement, l'intérêt du consommateur est pris en compte, dans la mesure où les droits de propriété intellectuelle qui ont pour but de protéger le fruit de la recherche, de l'invention ou de la création artistique permettent la création de marchandises, de produits ou de technologies qui sont utilisés dans la vie quotidienne. La musique, les logiciels d'ordinateurs, les films, les téléphones portables doivent leur commercialisation et leur utilisation possible, grâce à l'existence de droits de propriétés intellectuelles. Ainsi, ces droits revêtent une importance non seulement pour le titulaire des droits mais aussi pour le consommateur.

Dans son article sur le conflit entre les indications géographiques et les marques, Irina Kireeva, Avocate du cabinet O'Connor and Company, tente de marquer la distinction entre indications géographiques et marques, en analysant les arrêts de la Cour de justice des Communautés européennes (ci-après CJCE) les plus significatifs de la matière. Elle rappelle en introduction ce qui caractérise une indication géographique par rapport à une marque. En effet, les deux ont pour fonction d'identifier des produits, mais alors qu'une indication géographique est un signe utilisé sur des produits qui ont une origine géographique précise et possèdent des qualités, une notoriété ou des caractères essentiellement dus à ce lieu d'origine, une marque est un signe utilisé par une entreprise pour distinguer ses produits et ses services de ceux d'autres entreprises.

Pour les consommateurs, les indications géographiques servent à déterminer l'origine et la qualité des produits. Bon nombre d'entre elles ont acquis une renommée importante qui, faute d'être protégée de façon appropriée, peuvent faire l'objet de fausses déclarations de la part d'opérateurs commerciaux, préjudiciables aux consommateurs et aux producteurs légitimes. Les marques permettent quant-à elles au consommateur de choisir les produits qui leur donnent satisfaction et d'en écarter d'autres. Sans forcément lui reconnaître une qualité garantie, la marque permet donc au consommateur de s'attendre à un certain standard ou une constance dans la qualité des produits ou services.

Alors que les deux notions sont apparemment bien distinctes, les conflits entre indications géographiques et marques font l'objet de nombreux litiges tant aux niveaux international que communautaire. Il convient notamment de citer ici la multitude d'arrêts rendus par la CJCE dans des affaires impliquant toujours les mêmes protagonistes, Budéjovický Budvar et Anheuser-Busch dont un des dernier épisode est commenté à la fin de cette édition, dans la partie réservée au «Case Law of the Community Courts ».

La question de la prise en compte de l'intérêt du consommateur se pose avec acuité et suscite une vive controverse dans le domaine de l'innovation et du droit des brevets, ainsi que des affaires pendantes devant la Commission européenne (l'enquête sectorielle diligentée par la Commission européenne au sein des entreprises pharmaceutiques et de génériques en est un exemple frappant) et des arrêts récents de la CJCE (par exemple l'affaire Microsoft) le démontrent. Le titulaire d'un brevet bénéficie d'une protection pour une période limitée dans le temps qui lui permet 
de commercialiser le produit, fruit de son invention, ou de choisir les entités ou les personnes autorisées à commercialiser le produit. Une fois la période de protection échue, le produit tombe dans le domaine public et devient exploitable par d'autres, offrant ainsi un choix plus important pour le consommateur final dans la mesure où il pourra acquérir un produit similaire auprès d'entreprises concurrentes appliquant des normes de qualité et de prix différentes.

La question cependant de savoir si la période relativement longue de protection d'un brevet (20 ans en droit communautaire) peut avoir une incidence néfaste sur la concurrence, (donc pour le choix du consommateur) demeure ouverte et débattue. Alors qu'une partie de la doctrine pense que la protection absolue est injustifiée car elle constitue incontestablement un frein à l'innovation et à la création de produits nouveaux concurrents, les défenseurs de la propriété intellectuelle estiment au contraire que la protection se justifie par le fait que le créateur potentiel ne sera incité à créer, à investir et à dévoiler son invention que s'il sait qu'il pourra bénéficier d'une protection suffisamment longue contre la commercialisation de son invention par des tiers. Selon eux, la protection des droits ne met pas en péril les règles et les effets de la concurrence, dans la mesure où, par le biais de concessions de licences, de transferts de technologies et de pools de recherche, les droits du créateur peuvent cependant être utilisés par d'autres et ainsi inciter à la création de nouveaux produits ou nouvelles technologies.

Ce débat, loin d'être achevé, fait partie de l'analyse économique présentée par Matthew J. Elsmore dans son article critique sur la situation du brevet en Europe. L'auteur constate qu'une quantité impressionnante de demandes de brevets est déposée, instruite et acceptée en Europe et il se demande si une telle situation est satisfaisante tant d'un point de vue économique que qualitatif. Il fait ainsi part de ses doutes sur le point de savoir si le brevet constitue une véritable incitation à l'innovation ou non et si la protection par brevet est véritablement nécessaire en Europe. Si sa réponse est affirmative, il considère que des mesures doivent être prises pour améliorer le système européen afin que des brevets de meilleure qualité soient produits, ce qui engendrera une optimisation du bien public.

Un dernier domaine dans lequel le droit des consommateurs prend toute sa signification est celui du droit alimentaire. La politique de sécurité alimentaire de l'Union européenne qui vise à protéger la santé des consommateurs tout en garantissant le bon fonctionnement du marché intérieur établit des normes de contrôle en matière d'hygiène des denrées et produits alimentaires, de santé et de bien-être des animaux et de prévention des risques de contamination par des substances externes. Elle prescrit également des règles pour un étiquetage approprié de ces denrées et produits. Cette politique a été réformée au début des années 2000 conformément à l'approche dite « de la ferme à la table » qui vise à assurer un niveau élevé de sûreté des denrées et produits alimentaires commercialisés au sein de l'Union et offrir une garantie de qualité à toutes les étapes de la chaîne de production et de distribution.

Le renforcement de cette politique a justifié l'organisation par l'ERA de plusieurs conférences sur ce thème, notamment à Parme, au sein et en coopération avec l'Autorité européenne de sécurité des aliments. Lors de la dernière conférence organisée au mois d'octobre 2008, plusieurs récents développements législatifs du droit européen alimentaire ont été présentés et discutés, telles que les propositions législatives 
adoptées en 2006 portant sur les enzymes alimentaires, les arômes et les additifs, la nouvelle proposition de règlement sur les pesticides et la mise sur le marché des produits phytopharmaceutiques (Com (2006) 388 final) dont l'adoption est attendue pour le courant de l'année 2009 ou enfin, la proposition de règlement sur les limites maximales de résidus de pesticides dans les denrées alimentaires.

Ces dernières propositions qui modifient en profondeur la législation antérieure font l'objet, dans ce numéro, d'une contribution de Nicole Coutrelis, associée du Cabinet Coutrelis. Après avoir rappelé le fonctionnement du système antérieur applicable dans l'Union européenne, à savoir d'une part l'autorisation communautaire des substances actives puis la reconnaissance mutuelle et d'autre part, l'autorisation nationale des produits finis pour la mise sur le marché des produits phytopharmaceutiques (incluant les pesticides), l'auteur précise que le but général de la nouvelle réglementation sur les pesticides est de renforcer la protection de la santé des personnes et de l'environnement, tout en assurant un meilleur niveau d'harmonisation des procédures nationales d'autorisation et de reconnaissance mutuelle. Les substances chimiques jouent un rôle important lors de la production et de la consommation des produits alimentaires, tant pour l'agriculture et l'environnement que pour l'industrie agroalimentaire et enfin pour le consommateur.

\section{Le droit de la concurrence}

La protection de l'environnement ne joue pas seulement un rôle important dans le cadre du droit des consommateurs, mais aussi dans le droit des aides d'État. Si le droit de la concurrence dont le droit des aides d'État fait partie peut viser également à rendre la protection de l'environnement plus efficace, c'est aussi dans l'intérêt du consommateur. Ainsi le droit des aides d'État contribue à la protection des consommateurs. À la différence de la protection des consommateurs, il y un conflit qui soustend la relation entre protection de la concurrence et protection de l'environnement. Ce conflit est abordé devant les juridictions communautaires dans deux affaires récentes. Pieter van der Vegt, avocat chez PricewaterhouseCoopers, donne une analyse d'un arrêt du Tribunal de première instance des CE, portant sur le système d'échange de droits d'émission pour les oxydes d'azote au Pays-Bas, et d'un arrêt de la CJCE, portant sur la taxe environnementale sur les granulats au Royaume-Uni. Pour l'auteur, ces deux affaires illustrent parfaitement les difficultés résultant de l'application des dispositions du Traité CE sur les aides d'États aux mesures environnementales. L'auteur est en faveur d'une certaine autonomie des États membres quant à l'adoption de mesures environnementales tout en les soumettant au contrôle de sélectivité de l'aide en conformité avec l'article 87, paragraphe 1, du traité CE. Toutefois, le conflit entre protection de la concurrence et protection de l'environnement s'inscrit, aux yeux de l'auteur, dans un conflit plus large entre, d'une part, le principe de concurrence loyale et, d'autre part, la souveraineté des États membres. C'est pourquoi il analyse l'impact des arrêts dans ce contexte plus large. L'étude des deux arrêts a fait partie des discussions lors du $12^{\mathrm{e}}$ Forum sur les aides d'État organisé mi-novembre 2008 à Trèves.

De ce séminaire est également issu l'article de Jacques Derenne, associé chez Lovells, et Cédric Kaczmarek, sur le rôle du juge national dans la récupération des 
aides illégales. Tandis qu'à l'origine, la Commission et les États membres étaient au centre des procédures de récupération des aides illégales, le juge national et les tiers intéressés (nullo actore, nullus iudex) se sont vus, au fil du temps, attribués un rôle de plus en plus important. En 2005, la Commission en est arrivée à cette conclusion dans son Plan d'action dans le domaine des aides d'État où elle a rappelé que l'article 88, paragraphe 3, du traité $\mathrm{CE}$ a un effet direct et habilite les juges nationaux à récupérer à titre provisoire les aides illégales. Par conséquent, son souhait a été d'admettre les recours de droit privé devant les juridictions nationales, afin de « renforcer la discipline en matière de respect de la réglementation sur les aides d'État ». En ce qui concerne le tiers intéressé, les auteurs regrettent que des règles complexes et une multitude de voies de droit n'aient pas encore permis à ces acteurs de sortir de leur rôle marginal dans la procédure de récupération des aides illégales. Tout en louant les deux communications de la Commission, la première portant sur les principes relatifs à une mise en œuvre effective des décisions de la Commission, datant d'octobre 2007, la deuxième datant de février 2009 et portant sur l'application de la législation sur les aides d'État par les juridictions nationales, les auteurs sont d'avis qu'un «private enforcement » ne sera pas efficace tant que les voies de droit nationales ne seront pas harmonisées. Cependant, ils estiment qu'une telle revendication pourrait rencontrer des obstacles de la part des États membres dont une telle réforme dépendra; réforme dans un domaine où ces mêmes États membres sont aussi responsables des violations (Ovem lupo committere).

\section{La fiscalité transfrontalière}

La politique fiscale au sein de l'UE représente un élément essentiel du marché intérieur qui influence toutes les libertés économiques fondamentales du Traité. C'est notamment le cas des mesures qui facilitent le développement transnational des entreprises, l'épargne et bien sûr la consommation.

La coordination entre l'UE et les Etats membres devient essentielle pour cet instrument de régulation économique qui peut devenir un obstacle pour tout ce qu'il est appelé à développer. Cette fonction de régulation économique entraîne non seulement un besoin d'adaptation des systèmes fiscaux nationaux en vue de prendre en compte l'évolution du marché intérieur mais aussi la création de nouvelles structures sociétaires et de commerce.

Une des préoccupations communes les plus importantes se concentre sur le besoin d'éliminer les entraves aux opérations transfrontalières des entreprises, et par définition, les obstacles fiscaux qui se produisent entre entreprises qui sont établies dans le territoire du marché unique et qui veulent profiter des avantages fournis par celui-ci.

Dans ce domaine, le Conseil avait déjà adopté en 1990 deux directives qui visaient à éliminer les obstacles à cette coopération transfrontalière (la directive « Fusions » (90/434/CEE) et la directive «Sociétés mères-filiales » (90/435/CEE)). En complément, une convention a été conclue par les Etats membres (94/436/CEE) qui avait pour but d'introduire une procédure d'arbitrage relative à l'élimination des doubles impositions en cas de correction des bénéfices d'entreprises associées.

Cette convention d'arbitrage a marqué le début d'une politique fiscale relative aux prix de transfert à la suite de laquelle, dans le cadre de la Communication de 2001 
«Vers un marché intérieur sans entraves fiscales » (COM (2001) 582 final), un forum conjoint de l'UE sur les prix de transfert a été crée et, à l'issue des travaux du forum, un code de conduite pour la mise en oeuvre effective de la convention d'arbitrage a vu le jour.

Toutes les difficultés liées à ce domaine complexe dans la perspective du marché financier ont été soulevées lors de la conférence annuelle que l'ERA a organisée sur les récents développements du droit bancaire et financier à la fin de l'année 2008.

Les aspects fondamentaux de fiscalité concernant certains sujets relatifs aux marchés financiers méritaient d'être soulevés, comme l'impact du nouveau régime de TVA applicable aux services financiers et notamment, les défis relatifs aux prix de transfert relatif aux fusions et acquisitions transfrontalières de banques.

La contribution de David Ledure, Directeur de PricewaterhouseCoopers, et de Mourad Chatar, consultant chez PWC, développe ce dernier sujet. Elle met en exergue l'importance de la documentation pour pouvoir accréditer la nature des transactions réalisées entre entités liées et le respect du principe de pleine concurrence selon lequel les transactions commerciales et financières internationales entre sociétés liées doivent se conclure dans des conditions de pleine concurrence, au même titre que les transactions réalisées entre entités indépendantes (Arm's Lenght Principle).

Étant donné la disparité existante entre les Etats membres à propos de la documentation requise pour contrôler le respect de ce principe dans l'élaboration et la mise en oeuvre des prix de transfert, il y avait une demande de la part des entreprises de remédier à cette situation pour atteindre plus de sécurité juridique et de flexibilité. Ainsi, le Conseil, en juin 2006, avait adopté un code de conduite sur la documentation des prix de transfert pour les entreprises associées au sein de l'Union européenne (EUTPD) qui vise à harmoniser la documentation que les entreprises multinationales doivent fournir aux autorités fiscales au sujet de la méthode de fixation des prix de transfert qu'elles utilisent pour leurs transactions intra groupes transfrontalières.

Le contenu de ce code de conduite est examiné, ainsi que les règles concernant l'attribution de bénéfices aux établissements stables et son intérêt pour les banques. Finalement, et compte tenu de l'importance dans la situation financière actuelle, des allocations de risques pour les restructurations d'entreprises, une attention est portée au récent projet de rapport sur les aspects prix de transfert des restructurations d'entreprises et son impact (publié en septembre 2008).

Si la Commission est consciente du besoin d'éliminer les obstacles fiscaux au sein du marché intérieur, il en va de même pour la CJCE. Pour mieux comprendre l'importance au sein du marché intérieur de l'impact de la fiscalité dans le développement des activités transnationales des fondations, l'ERA a organisé dans sa représentation bruxelloise, une conférence-«briefing » pour réfléchir à l'impact de l'arrêt de la CJCE du 27 janvier 2009 (C-318/07; Hein Persche c. Finanzamt Lüdenscheid).

L'expansion et l'impact économique du secteur des fondations de ces 15 derniers années, oblige les fondations à trouver des instruments appropriés pour opérer de façon effective dans tout le territoire du marché intérieur. Même si aujourd'hui il est évident que les fondations se voient octroyées par le Traité la possibilité de se déplacer au sein de l'UE, il n'est pas contesté qu'elles sont confrontées à un nombre important d'obstacles pour pouvoir mettre en place leurs activités transfrontalières. Ainsi, quelques-uns des systèmes fiscaux nationaux ont été à l'origine du traitement 
discriminatoire dont étaient l'objet des fondations non-résidentes, face au traitement fiscal octroyé aux fondations résidentes.

Au niveau européen, les différents acteurs sont conscients du problème et de la nécessité de réagir en conséquence. Au niveau politique, cette réalité était déjà inscrite comme une priorité dans le Plan d'Action de la Commission européenne de 2003 (COM (2003) 284 sur la « Modernisation du droit des sociétés et renforcement du gouvernement d'entreprise dans l'Union européenne - Un plan pour avancer») qui a été récemment suivi par la publication d'une étude de faisabilité, à la demande de la Commission, sur la création éventuelle d'un statut de la fondation européenne, actuellement en cours de consultation.

Au niveau jurisprudentiel, en 2006, la Cour avait déjà eu l'occasion de se prononcer sur ce sujet dans l'affaire Stauffer (C-386/04 ; Centro di Musicologia Walter Stauffer c. Finanzamt München für Körperschaften) dans laquelle elle avait conclu à l'application du principe de non-discrimination en ce qui concerne les bénéfices fiscaux octroyés aux fondations, sans faire de distinction selon le lieu de résidence. Récemment, la Cour a eu une nouvelle opportunité de se prononcer dans l'affaire Persche où elle s'est penchée sur la déductibilité fiscale des dons transfrontaliers versés à des organismes établis et reconnus d'intérêt général dans un autre Etat membre, et sur sa compatibilité avec le traité, notamment avec la libre circulation des capitaux.

L'ERA, également consciente de l'importance de ce sujet pour les praticiens, a voulu offrir un forum de débat dont la différente provenance professionnelle des experts présents dans le briefing a permis de réfléchir sur l'impact de l'arrêt Persche pour le développement des activités transnationales des fondations et sur les différentes actions en cours au niveau européen pour favoriser la prise en compte des bénéfices du marché unique pour les fondations.

Annette Deckers, du Ministère des finances des Pays-Bas, Hanna Surmatz, experte du European Foundation Center (EFC) et Thomas von Hippel, ancien chercheur au Max-Planck-Institut de droit privé international et comparé et actuellement juge à Hambourg, ont fourni l'occasion d'un débat vif et intéressant.

Ce débat, ainsi que les principaux aspects relevés par l'arrêt font l'objet de la contribution fournie par Thomas von Hippel à ce numéro d'ERA Forum. Il mène le lecteur à travers les différentes étapes de l'établissement par la CJCE du principe de non-discrimination. Il explique comment cette consolidation a entraîné également des conséquences importantes, telle que le test de comparabilité, et comment celui-ci peut nuire à l'exercice des libertés fondamentales par les fondations, étant donné que son application, et par définition le respect du principe de non-discrimination, revient aux Etats membres, par le biais de leurs systèmes fiscaux ou contentieux. Il illustre la difficulté de cette application, sous l'angle du système fiscal allemand, en testant la fondation concernée dans l'affaire Stauffer. L'auteur ne se limite pas à mettre en exergue les problèmes de cette mise en œuvre, mais il se penche également sur des options pour y remédier qui rejoignent d'ailleurs dans une majorité, les propositions retenues par l'étude de faisabilité susmentionnée.

Bonne lecture ! 